\title{
Scan Projection Optical Lithography through a Slit onto Small Diameter Shafts
}

\author{
Toshiyuki Horiuchi and Naoki Hayashi* \\ Tokyo Denki University \\ Graduate school of Engineering, Tokyo Denki University \\ 2-2, Kanda-Nishiki-cho, Chiyoda-ku, Tokyo, 101-8457, Japan \\ *Present Address: Canon Inc.
}

\begin{abstract}
Scan projection optical lithography onto small diameter shafts were investigated. It will be useful for printing complicated patterns including cross points or elbow points instead of laser direct writing. A specimen shaft coated with a resist film was synchronously rotated during the continuous linear scan of a flat reticle. Because the shaft surface was curved in an arc, instantaneous exposure field should be limited to a narrow area near the top surface of the shaft, where the surface was regarded almost flat. Accordingly, an allowable width of the slit limiting the exposure area was discussed considering defocus, pattern position shift, and width swelling. Pattern width target was $50-100 \mu \mathrm{m}$ onto 1 or 2 mm diameter shafts because an immediate application was to print helical shaft patterns for micro-axial pumps. The slit width had to be less than approximately $30 \%$ of the shaft diameter to print $100-\mu \mathrm{m}$ wide patterns clearly. The cross patterns were printed as clearly as the simple line patterns, and width and pitch variations were sufficiently small.
\end{abstract}

Keywords: projection lithography, fine shaft, scan exposure, slit width, cross pattern

\section{Introduction}

Lithography is widely used for fabricating micro-electro-mechanical systems (MEMS). For this usage, it is often required to form minute patterns onto not only usual flat substrates but also shaft and pipe surfaces. It is difficult to print patterns onto steeply curved surfaces. However, various lithography methods such as direct writing using a laser beam $[1,2,3]$, X-ray proximity printing [4-7], and optical projection exposure $[8,9]$ have already been developed. Even onto inside surfaces of fine pipes, minute patterns with widths of 10-200 $\mu \mathrm{m}$ have been delineated $[10,11]$.

Direct writing is superior in arbitrariness and minuteness. However, it takes a long time to delineate complicated patterns. Besides, it is very difficult to make the pattern widths uniform at cross points and elbow corners, because the scanned at the cross or turning points. From these points of view, optical projection lithography is favorable and promising. Since reticle patterns in an exposure field are replicated at a time, exposure time does not depend on the complicatedness and density of patterns. Besides, the cross points and elbow corners are exposed by the same condition with other simple line parts. Accordingly, stitching errors and over dose exposures caused by the double exposure are avoided. Though the X-ray proximity lithography has the same merit, it is cost-worthy and not commonly available.

For this reason, optical projection lithography onto shafts with 1-2 $\mathrm{mm}$ is investigated here. Patterning onto such shafts is useful to crave fluid bearing grooves, pump spirals and others. 


\section{Projection Exposure onto Fine Shafts}

Figure 1 shows the principle of optical projection exposure investigated here. A usual flat reticle was linearly scanned synchronizing to the rotation of a specimen shaft to be exposed. Since the exposure shaft has a round shape at the top, the exposure area was limited to an extent where the shaft top is considered to be approximately flat. If the slit width is too large, exposed surface height of the shaft changes at both peripheries of the exposure field, and the projected pattern images are variously defocused depending on the curvature.

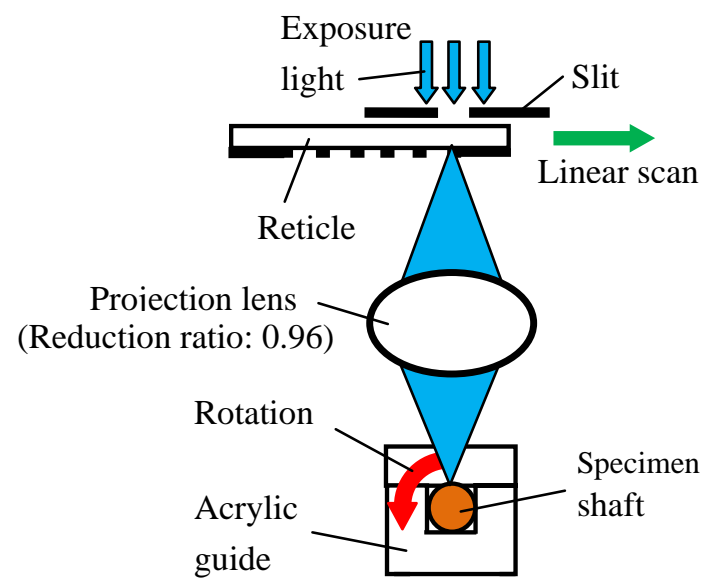

Figure 1. Principle of optical projection exposure onto fine shafts

Patterns printed on the specimen surface have to be stitched exactly smooth when the shaft is rotated around the axis. Accordingly, the shaft circumference length or the shaft diameter has to be strictly examined so as they exactly correspond to the reticle scan length.

The slit width was decided considering the defocus caused by the curvature of the shaft circumference in the exposure field. The maximum defocus error $\Delta h$ at the slit image boundary is shown in Fig. 2. When a half width of the slit image is $s$, and the shaft radius is $r$, defocus error $\Delta h$ is shown by the following equation.

$$
\Delta h=r-r \cos \varphi .
$$

Here,

$$
\sin \varphi=\frac{s}{r}
$$

By substituting eq. (2) to eq. (1),

$$
\Delta h=r-r \sqrt{1-\frac{s^{2}}{r^{2}}}=r-\sqrt{r^{2}-s^{2}} .
$$

The defocus errors calculated for radiuses of 0.5 and $1 \mathrm{~mm}$ are shown in Fig. 3.

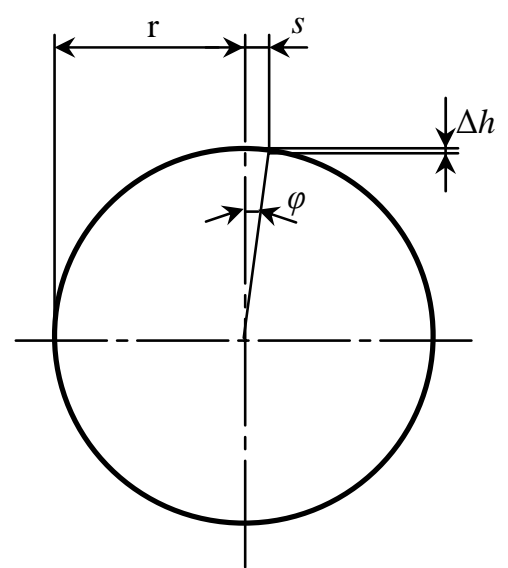

Figure 2. Defocus caused by the circular surface

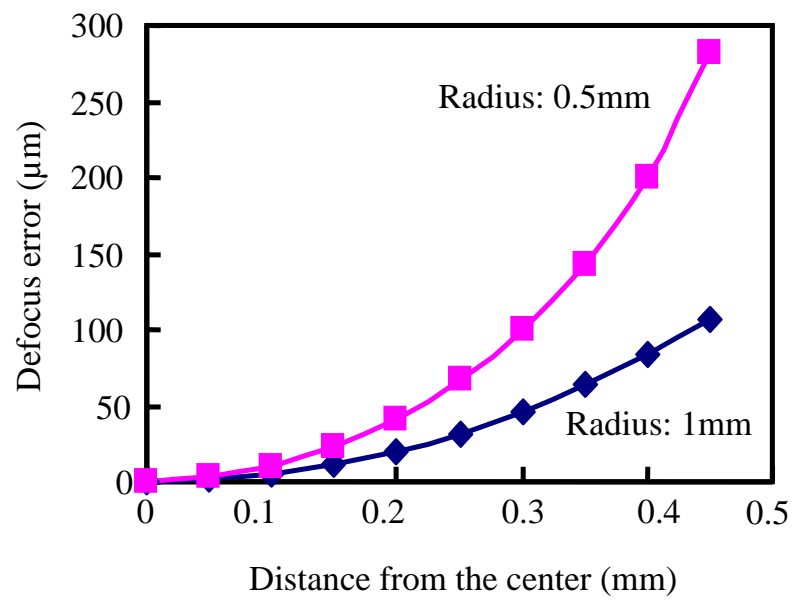

Figure 3. Defocus error caused by the shaft surface curvature

On the other hand, when periodical patterns on a reticle are illuminated by light rays perpendicular to the reticle, the first order diffraction angle $\theta$ is obtained by the following equation.

$$
\theta=\sin ^{-1} \frac{\lambda}{p} .
$$

Here, $\lambda$ is the exposure wavelength of $436 \mathrm{~nm}$, and $p$ is the pattern pitch.

The first order diffraction light rays with an angle of $\theta$ are collected with an angle of $\theta$ ' on the 
wafer and form pattern images, as shown in Fig. 4. When the magnification ratio is $m$,

$$
m \sin \theta^{\prime}=\sin \theta .
$$

From eqs. (4) and (5),

$$
\sin \theta^{\prime}=\frac{1}{m} \sin \theta=\frac{\lambda}{m p} \text {. }
$$

When the blur region is $b$ at the position being $d$ distant from the focused point, $d$ is calculated by the following equation.

$$
d=\frac{b}{\tan \theta^{\prime}}=\frac{b \cos \theta^{\prime}}{\sin \theta^{\prime}}=\frac{b \sqrt{1-\sin ^{2} \theta^{\prime}}}{\sin \theta^{\prime}} .
$$

Substituting eq. (6) for $\sin \theta$ ' in eq. (7),

$$
d=b \sqrt{\left(\frac{m p}{\lambda}\right)^{2}-1} .
$$

When $b=p / 2$ and $m=1$,

$$
d=\frac{p}{2} \sqrt{\left(\frac{p}{\lambda}\right)^{2}-1} .
$$

Accordingly, the depth of focus (DOF) for periodical patterns with a width or a half pitch of $p / 2$ is calculated as

$$
D O F=2 d=p \sqrt{\left(\frac{p}{\lambda}\right)^{2}-1} .
$$

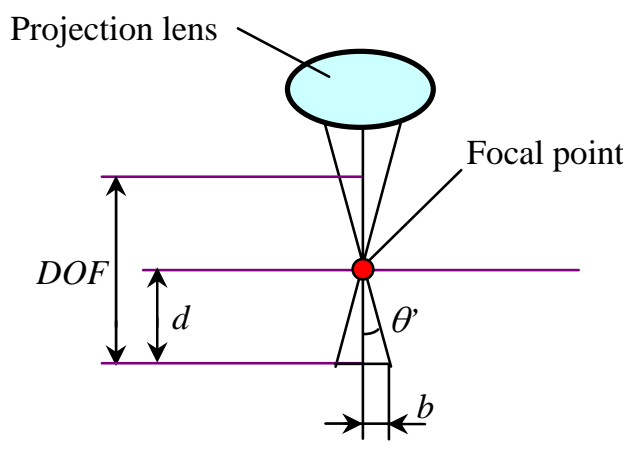

Figure. 4. Explanation of depth of focus

DOF calculated by eq. (10) is shown in Fig. 5 . The change at the medium appears considering the blurs caused by the second order diffraction light rays. The $D O F$ values are sufficiently larger than the defocus error calculated and shown in Fig. 3

On the other hand, pattern lengths become long because the shaft surface inclines depending on the curvature. When a half width of the slit is $s$, pattern length elongation $\Delta L$ printed on the arc $\mathrm{AB}$ in Fig. 6 is expressed as

$$
\Delta L=r \varphi-s=r \sin ^{-1} \frac{s}{r}-s .
$$

Calculated $\Delta L$ for various $s$ is shown in Fig. 7.

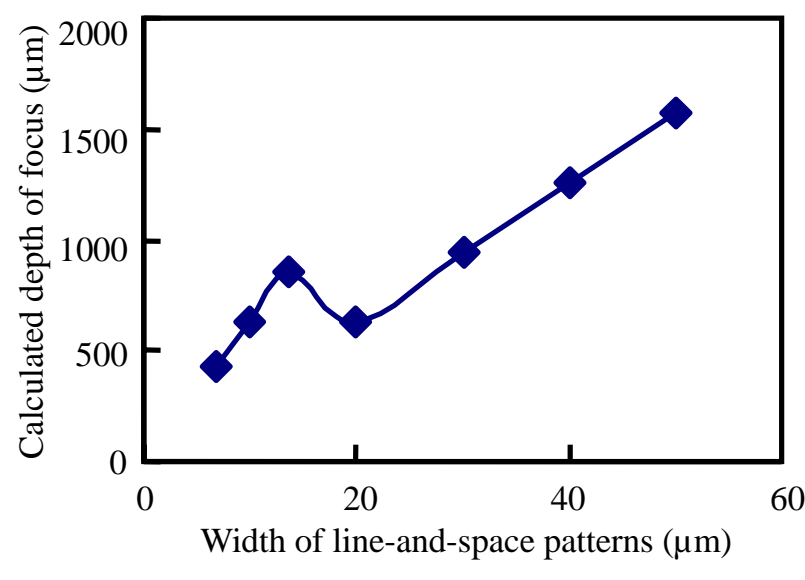

Figure 5. Calculated depth of focus

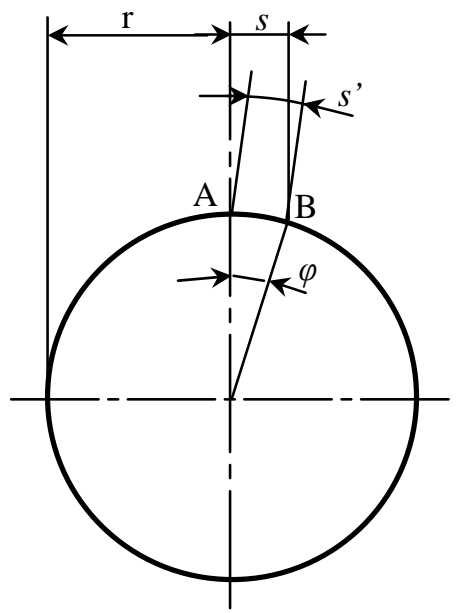

Figure 6. Explanation of pattern length elongation

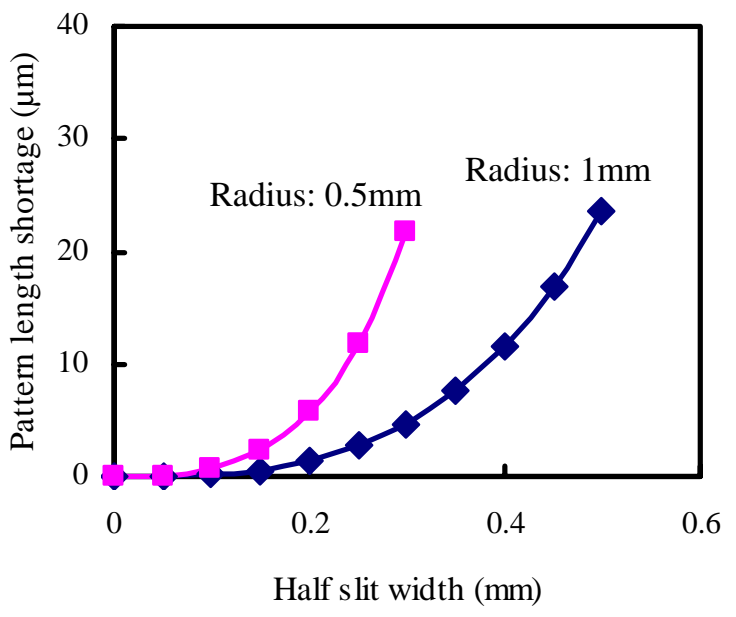

Figure 7. Pattern length elongation corresponding to the shaft surface curvature 
In addition, referring to Fig. 8, pattern width swelling $\Delta w$ for patterns with a width of $w$ near the slit edge is calculated by

$$
\Delta w=w\left(\frac{1}{\cos \varphi}-1\right) \cong w(1-\cos \varphi) .
$$

Calculation results of the pattern width swelling $\Delta w$ are shown in Fig. 9.

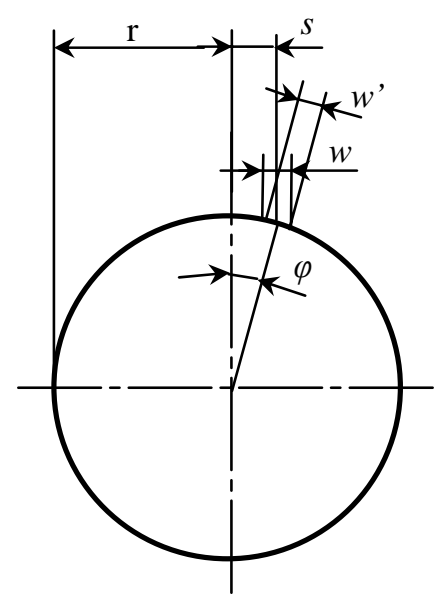

Figure 8. Explanation of pattern width swelling

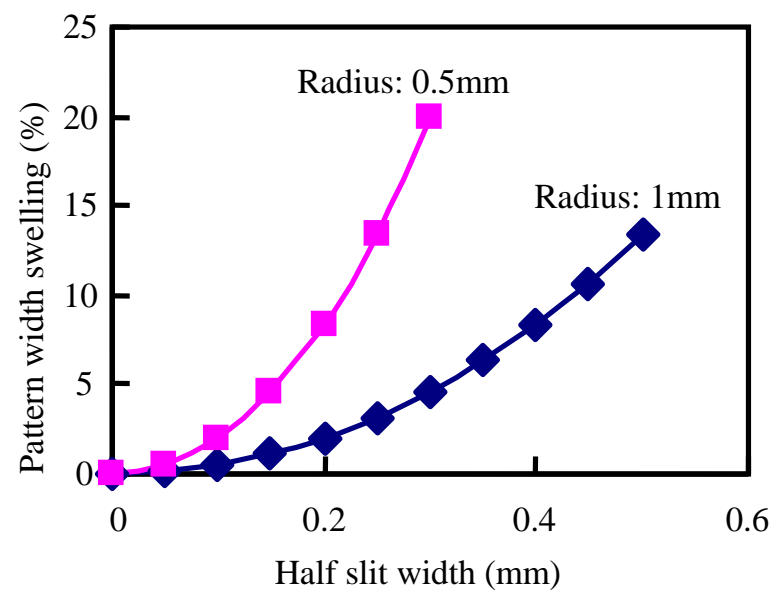

Figure 9. Calculated pattern width swelling

The circumference lengths of specimen shafts should be exactly equal to the scan length of the reticle. However, the specimen shaft diameters have deviation, the cross sections are not genuine circles, and the rotation center does not exactly identical with the specimen centers. In addition, the stitching error at $0^{\circ}$ and $360^{\circ}$ positions on the circumference is somewhat affected by the inertial responses of the reticle scan and specimen rotation stages at the start and stop moments.

However, if the exposure position on the specimen circumference is controlled by the rotation angle of the specimen shaft, the linear scan time of the reticle and the rotation time of the specimen shaft are able to be adjusted almost exactly the same. For this reason, patterning blurs are mainly caused by above $\Delta L, \Delta w$ and the difference between the rotation center and the specimen center, if the specimen diameters are strictly managed to be almost exactly same.

\section{Exposure Experiments}

Experiments to investigate the influence of the slit width were executed using a handmade test exposure system shown in Fig. 10. As an exposure source, a super high-pressure mercury lamp with a power of $250 \mathrm{~W}$ was used, and the light rays were filtered by a 436-nm-wavelength band pass filter. The projection lens was a camera lens, and the F-number was set at 8 , which corresponds to the numerical aperture (NA) of 0.031. Though the magnification of the projection lens was adjusted to approximately 1 , actually measured one was 0.96 . The system has the size of $350 \mathrm{~mm}$ width, $336 \mathrm{~mm}$ depth and $700 \mathrm{~mm}$ height.

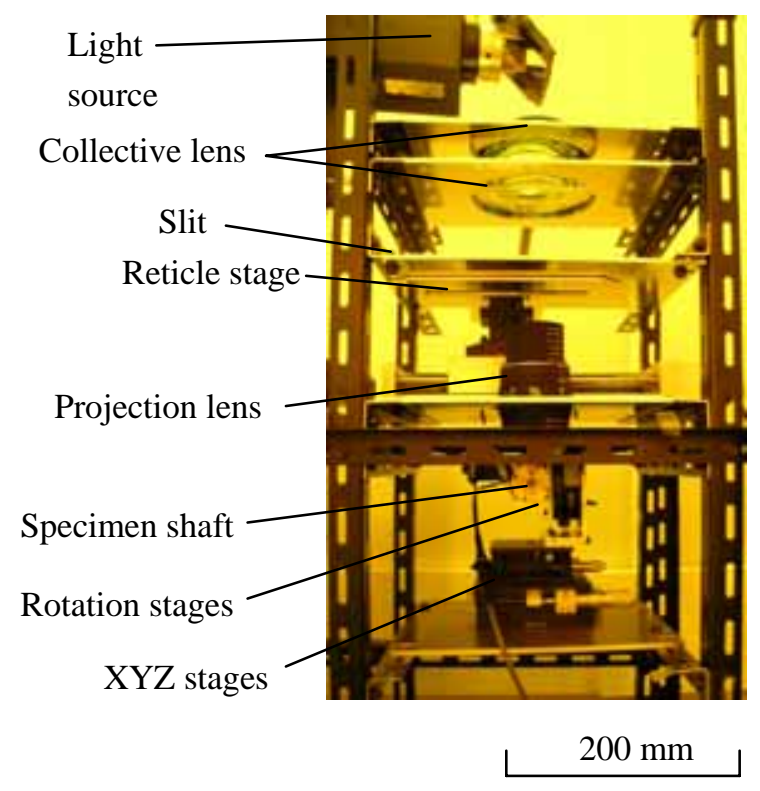

Figure 10. Hand-made exposure system used in the experiments

Copper specimen shafts with diameters of 1 and $2 \mathrm{~mm}$ (radius of $0.5 \mathrm{~mm}$ and $1 \mathrm{~mm}$ ) were 
prepared, and they were coated with positive resist films. PMER P-LA900 (Tokyo Ohka Kogyo) was used as a resist. To coat the resist, each specimen shaft was vertically held by a small chuck, and it was sunk into the bottled resist. When the specimen shaft was relatively drawn up from the resist by moving the resist bottle downward, it was uniformly coated by the resist film. The resist film thickness was controlled by changing the relative draw-up speed, as shown in Fig. 11. In the following experiments, the resist thickness was kept approximately $5 \mu \mathrm{m}$, and the resist was coated in 20-mm region from the shaft tip. The specimen shafts were pre-baked in an electric oven at $85^{\circ} \mathrm{C}$ for one hour.

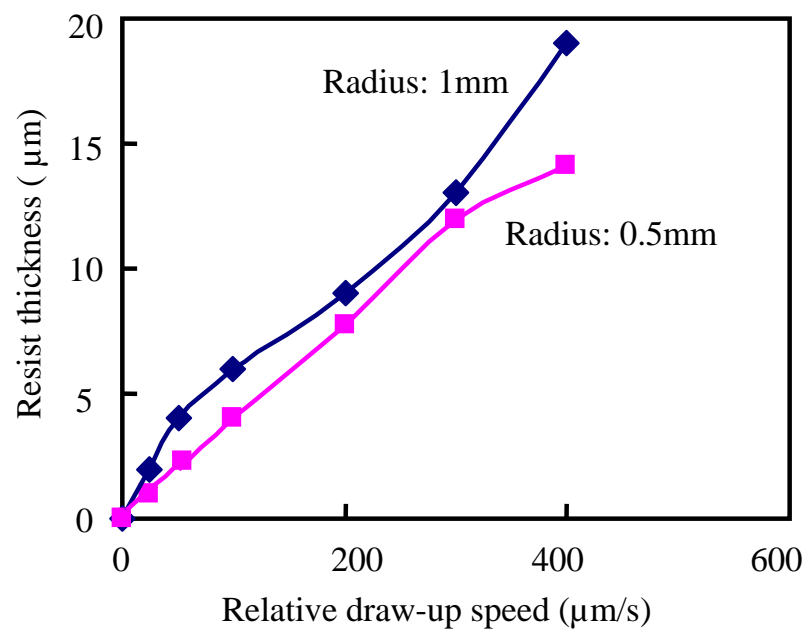

Figure 11. Resist thickness variation when changing the relative draw-up speed

Influence of the slit width was investigated at first by printing cross check patterns with 100- $\mu \mathrm{m}$ space and 500- $\mu \mathrm{m}$ pitch onto 2-mm diameter specimen shafts. Figure 12 shows the replicated patterns observed at the stitching angles for various wide slits. The pattern profiles were almost same when the slit width was less or equal to $0.6 \mathrm{~mm}$. However, the pattern edges became unclear if the slit width was more than $0.7 \mathrm{~mm}$. Next, the same patterns were printed on 1-mm diameter specimen shafts. When a slit with a width of $0.2 \mathrm{~mm}$ was used, patterns were printed almost comparably to the ones printed on 2-mm diameter shafts using a slit with a width less than or equal to $0.6 \mathrm{~mm}$. The result is shown in Fig. 13.

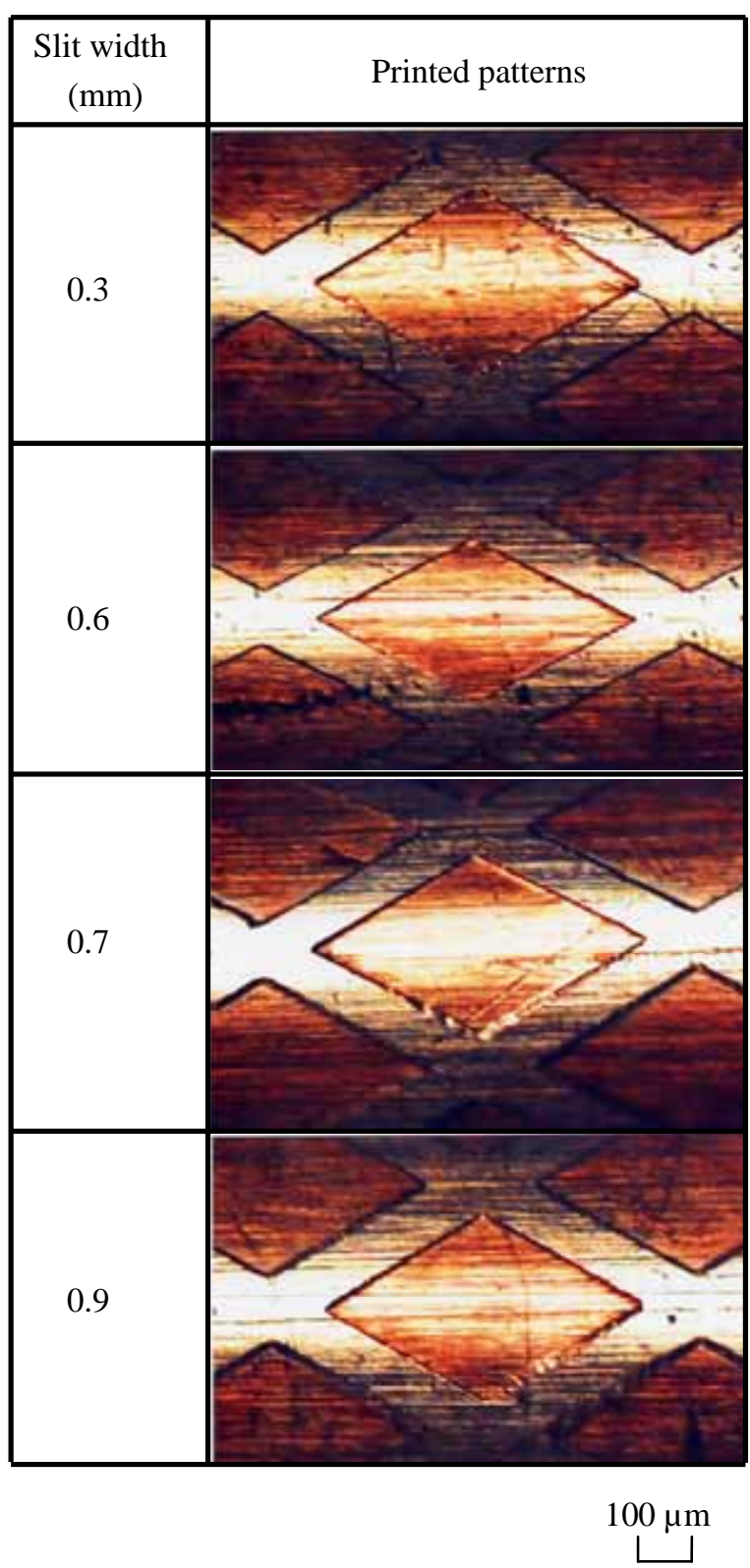

Figure 12. Patterns printed on 2-mm diameter shafts using various wide slits

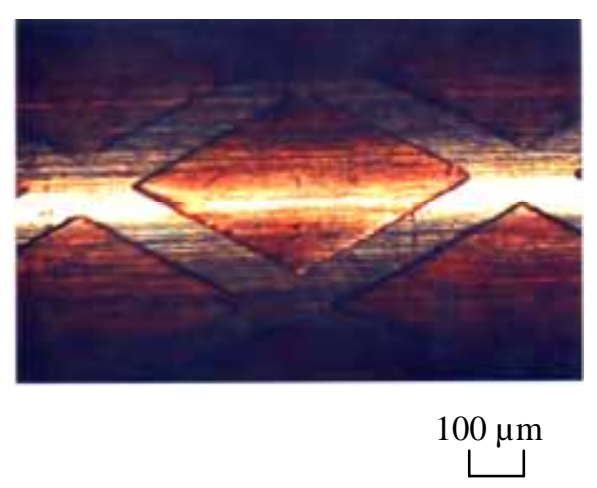

Figure 13. Patterns printed on a 1-mm diameter specimen shaft using a slit with a width of $0.2 \mathrm{~mm}$ 
From the calculation results shown in Fig 9, pattern width swelling at the slit periphery becomes approximately $5 \%$ of the pattern width when the slit width was $0.6 \mathrm{~mm}$ for a $2-\mathrm{mm}$ diameter specimen shaft. Pattern edge blurs shown in Fig. 12 seem being in reasonable ranges.

To investigate minutely, pattern widths and pitches were measured for specimen shafts with 2-mm diameter. Pattern widths and pitches were measured on the circumferences by $60^{\circ}$ interval. Variations for the 6 measured widths and pitches are shown in Figs. 14 and 15, respectively. The width variation became gradually large according to the increase of the slit width. This is because the pattern length elongation and width swelling are enhanced according to the increase of the slit width, and the shifted pattern image intensity distributions

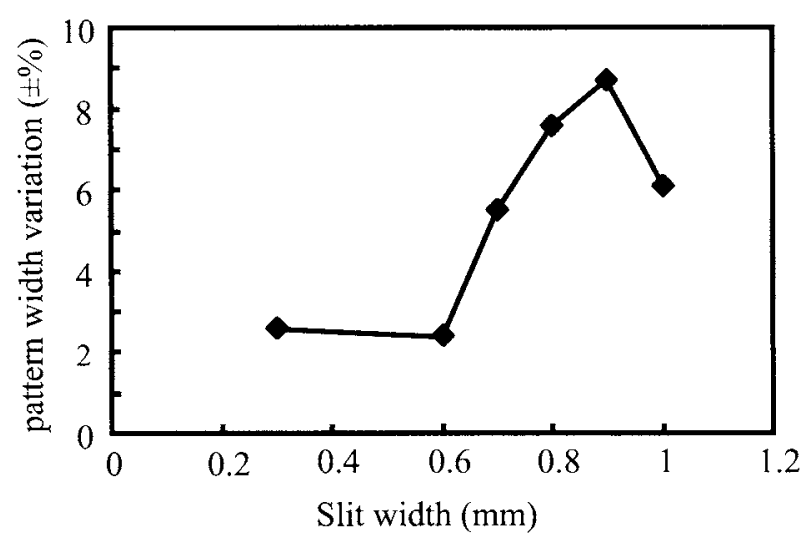

Figure 14. Relationship between pattern width variation and slit width

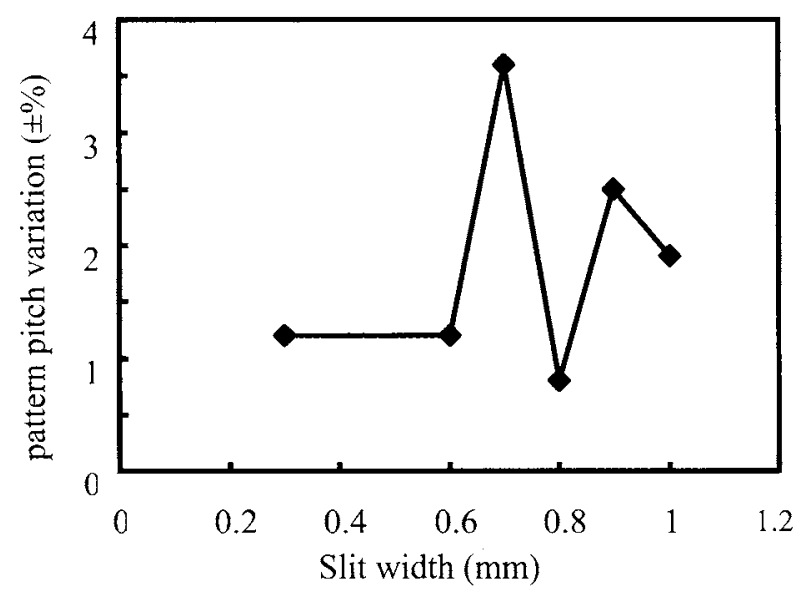

Figure 15. Relationship between pattern pitch variation and slit width are superimposed during the scanning. Because the specimen shafts were rotated and continuously exposed at all the positions in the slit area, the pattern length elongation and the width swelling were averaged, and the pattern widths were not directly changed corresponding to the pattern length elongation and the width swelling, but they were averagely changed depending on these phenomena.

On the other hand, however, pattern pitch variations were fairly small and did not almost depend on the slit width. This is probably because the pattern degradation using the wide slits almost equally arose at both pattern sides.

By the way, in all the experiments, cross check patterns were printed without over-dose at cross points, and the crossed corner profiles were as sharp as the straight line parts. It was confirmed that the projection exposure had a remarkable advantage for printing such patterns including cross points.

\section{Conclusion}

From the geometrical analyses and various experiments, influences of the slit width in the optical projection lithography onto fine cylindrical shafts were discussed. The slit width had better be as narrow as possible if only the patterning accuracy was considered without thinking the exposure throughput. However, the narrow slit brings the elongation of the exposure time. Therefore, appropriate slit width should be investigated and decided.

As a result of this research, it was clarified that the pattern profiles did not almost degrade if the slit width was not too large. The slit width limits were approximately $0.6 \mathrm{~mm}$ for $2 \mathrm{~mm}$ diameter specimen shafts. This limit corresponds to the slit width by which the maximum pattern width swelling in the slit becomes approximately $5 \%$ of the pattern width. The slit width seems reasonable because the width is approximately $30 \%$ of the specimen diameter and will be sufficiently wide for the practical use. It will be quite easy to reshape the exposure light rays efficiently suiting to this slit width. 


\section{Acknowledgement}

This work was partially supported by Research Institute for Science and Technology of Tokyo Denki University Grant Number Zc08-01 in 2008 and Q09M-05 in 2009.

\section{References}

1. Y. Joshima, T. Kokubo, T. Horiuchi: Jpn. J. Appl. Phys. 43 (2004) 4031-4035.

2. Y. Kaneko, K. Hashimoto, T. Horiuchi: Microelectronic Engineering, 83 (2006) 1249- 1252.

3. M. Katayama, T. Horiuchi: Jpn. J. Appl. Phys., 47 (2008) 4762-4764.

4. A. D. Feinerman, R. E. Lajos, V. White, and D. D. Denton: J. Microelectromechanical Sys., 5 (1996) 250-255.

5. T. Katoh, N. Nishi, M. Fukagawa, H. Ueno, S. Sugiyama: Proc., MEMS 13th Int. Conf., (2000) 556-561.
6. H. Mekaru, S. Kusumi, N. Sato, M. Yamashita, O. Shimada, T. Hattori: Jpn. J. Appl. Phys., 43 (2004) 4036-4040.

7. H. Mekaru, S. Kusumi, N. Sato, M. Shimizu, M. Yamashita, O. Shimada, T. Hattori: Jpn. J. Appl. Phys., 44 (2005) 5749-5754.

8. K. Hashimoto, Y. Kaneko, T. Horiuchi: Microelectronic Engineering, 83 (2006) 1312- 1315.

9. T. Horiuchi and N. Hayashi: Micro- electronic Engineering, (2009) doi:10.1016/j.mee. 2008.12.078.

10. T. Horiuchi, M. Katayama, Y. Watanabe, K. Fujita and T. Yasuda: Micro-electronic Engineering, 85 (2008) 1043-1046.

11. K. Saito and T. Horiuchi: Tech. Dig. Microprocess and NanoTechn., (2008) 460-461. 